\section{Anterior spinal cord infarction with permanent paralysis following endoscopic ultrasound celiac plexus neurolysis}

Celiac neurolysis is an effective adjunct for managing refractory pancreatic cancer pain. Endoscopic ultrasound (EUS) offers several potential technical advantages over the traditional percutaneous technique [1]. We report the first case of paraplegia following EUS celiac neurolysis.

A 76-year-old man underwent EUS celiac neurolysis for refractory pain secondary to unresectable pancreatic cancer. The major arteries all demonstrated normal pulse Doppler imaging, using a linear echoendoscope (UC140P-AL5; Olympus America, Center Valley, Pennsylvania, USA) ( Fig. 1). Several small celiac ganglia were noted. A 22-gauge needle was advanced into the largest ganglion, and $1 \mathrm{~mL}$ of alcohol (99\%) and bupivacaine $(0.25 \%)$ mixture was injected. Another $23 \mathrm{~mL}$ was injected into the celiac plexus. No immediate complications were noted.

Upon awakening from general anesthesia, the patient noted paralysis of his lower extremities. Emergent magnetic resonance imaging (MRI) of the thoracic and lumbar spine revealed an anterior spinal cord infarct from T10 to the conus medullaris in the distribution of the anterior spinal artery ( Fig. 2 ). Imaging also demonstrated T2 hyperintensity of the paraspinal musculature ( $\bullet$ Fig.3), suggesting some solution may have passed via the left T12 intercostal artery, which supplies both the spinal cord via a radiculomedullary artery and the paraspinal muscles via dorsal branches. The patient remained paraplegic until death 24 days later.

Percutaneous and EUS-guided celiac neurolysis relieves pain in $80 \%$ of patients with pancreatic cancer $[2,3]$. Major complications develop in $1 \%-2 \%$ of patients, and include lower extremity paraplegia, puncture of adjacent organs, and gastroparesis [2-4]. Neurologic complications develop secondary to ischemia or direct injury to the spinal cord or somatic nerves [5].

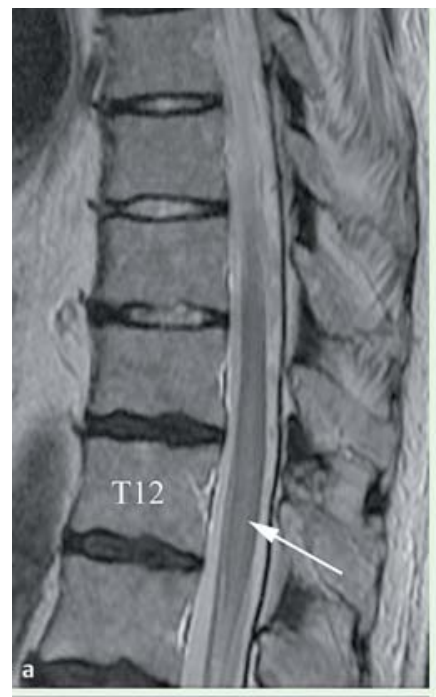

This patient highlights a rare but clinically significant risk of EUS celiac neurolysis.
Therefore, appropriate patient counseling and consent are key in moderating expectations of pain relief and conveying the risks of EUS celiac neurolysis, which include paralysis.

Endoscopy_UCTN_Code_CPL_1AL_2AF

\section{Competing interests: None}

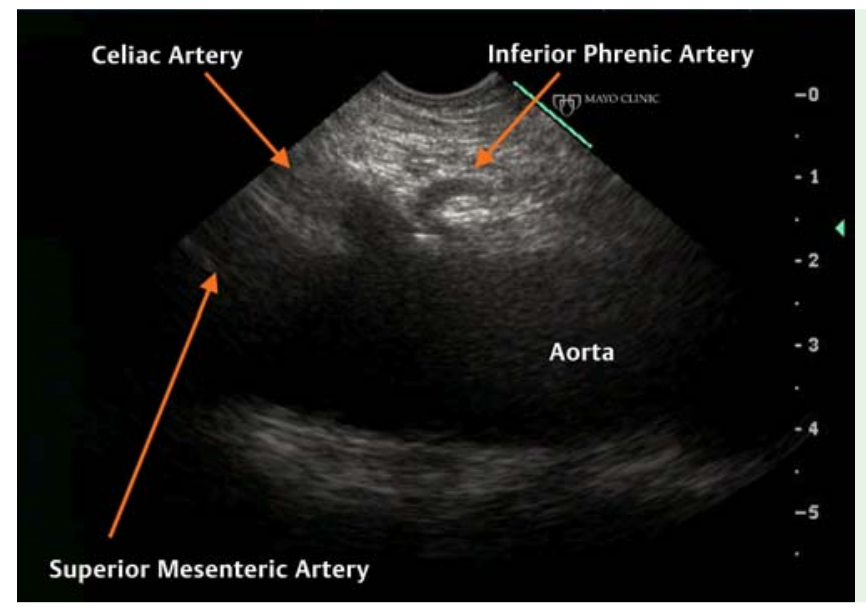

Fig. 1 Endoscopic ultrasound (EUS) imaging of the major arteries around the celiac plexus. Vascular structures including the celiac trunk, superior mesenteric artery, and inferior phrenic artery were all examined to help avoid inadvertent injection.

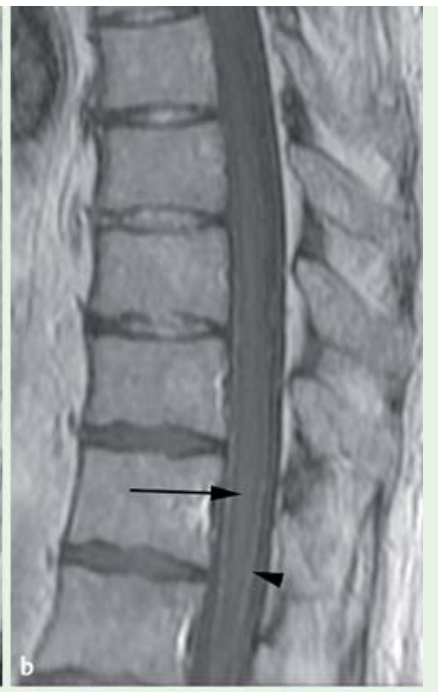

Fig. 2 Sagittal magnetic resonance imaging (MRI) demonstrating the spinal cord infarct. a Sagittal fast spin-echo (FSE) T2weighted imaging demonstrating diffuse intramedullary $\mathrm{T} 2$ hyperintensity throughout the lower thoracic cord and conus (white arrow). b Sagittal gadolinium-enhanced T1-weighted imaging demonstrating faint intramedullary enhancement (black arrow) with mild prominence of the pial vascularity (black arrowhead). c Sagittal diffusion-weighted images showing diffuse hyperintensity (white arrow) in the conus. d Sagittal apparent diffusion coefficient (ADC) map demonstrating decreased signal (white arrow) in the same region, consistent with true restricted diffusion and not T2 shine-through. 


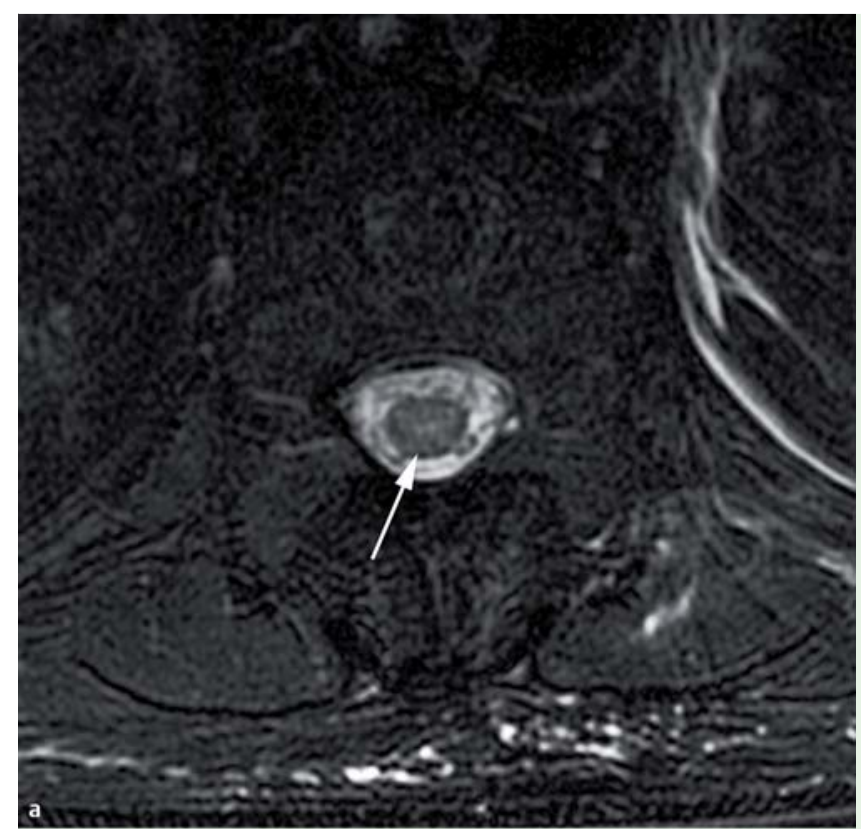

Fig. 3 Axial magnetic resonance imaging (MRI). a Axial fast spinecho (FSE) T2-weighted imaging with fat saturation demonstrating diffuse intramedullary T2 hyperintensity involving the grey and white matter (white arrow). b Axial FSE T2-weighted imaging, slightly inferior, also demonstrating $\mathrm{T} 2$ hyperintensity in the paraspinal musculature anteriorly and posteriorly (white arrowheads). Both of these muscle groups are supplied via the intercostal artery.

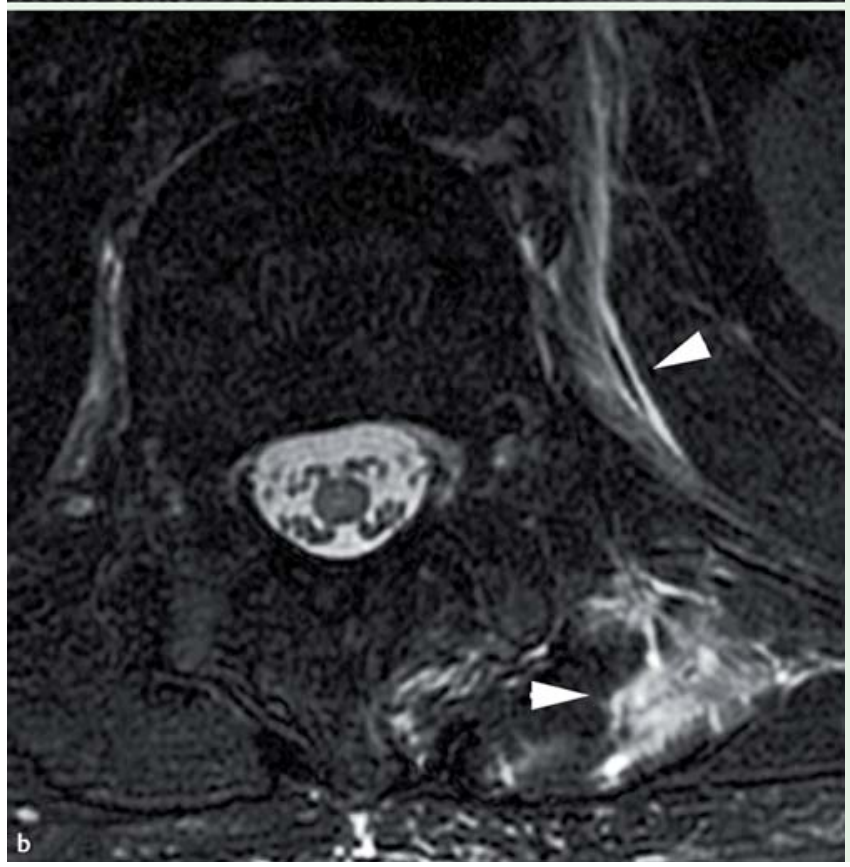

\section{Fujii ${ }^{1,2}$, J. E. Clain ${ }^{1,2}$, J. M. Morris ${ }^{1,2}$, M. J. Levy ${ }^{1,2}$}

${ }^{1}$ Division of Gastroenterology and Hepatology, Mayo Clinic, Rochester, Minnesota, USA

${ }^{2}$ Division of Neuroradiology, Mayo Clinic, Rochester, Minnesota, USA

\section{References}

1 Levy MJ, Wiersema MJ. Endoscopic ultrasound-guided pain control for intra-abdominal cancer. Gastroenterol Clin N Am 2006; 35: $153-165$

2 Eisberg E, Carr DB, Chalmers TC. Neurolytic celiac plexus block for treatment of cancer pain: a meta-analysis. Anesth Analg 1995; 80: $290-295$

3 Puli SR, Reddy JBK, Bechtold ML et al. EUSguided celiac plexus neurolysis for pain due to chronic pancreatitis or pancreatic cancer pain: a meta-analysis and systematic review. Dig Dis Sci 2009; 54: 2330-2337

4 Davies DD. Incidence of major complications of neurolytic coeliac plexus block. J R Soc Med 1993; 86: 264-266

5 van Dongen RT, Crul BJ. Paraplegia following coeliac plexus block. Anesthesia 1991; 46: $862-863$

\section{Bibliography}

DOI http://dx.doi.org/

10.1055/s-0032-1309708

Endoscopy 2012; 44: E265-E266

(c) Georg Thieme Verlag KC

Stuttgart · New York

ISSN 0013-726X

\section{Corresponding author}

\section{J. Levy, MD}

Division of Gastroenterology and Hepatology

Mayo Clinic

200 First Street SW

Rochester

MN 55905

USA

Fax: +1-507- 266-3939

levy.michael@mayo.edu 